\title{
Qualität und Sicherheit chinesischer Arzneidrogen in Deutschland - ein Update
}

\author{
Nadja Hempen Roman Huber \\ Uni-Zentrum Naturheilkunde, Institut für Umweltmedizin und Krankenhaushygiene, Universitätsklinikum Freiburg, Freiburg, Deutschland
}

\author{
Schlüsselwörter \\ TCM · Experte · Systematisches Review · \\ Unerwartete Nebenwirkung · Schwermetall · Pestizid . \\ Aflatoxin
}

\section{Zusammenfassung}

Hintergrund: Arzneimittel (TCA) der traditionellen chinesischen Medizin (TCM) hatten in der Vergangenheit zum Teil erhebliche Mängel bezüglich Qualität und Sicherheit. Der aktuelle Wissensstand hierzu wurde evaluiert. Material und Methoden: Experteninterviews mittels standardisierter Fragebögen, systematische Literaturrecherche für Studien aus Deutschland publiziert in 1998-2013, systematische Anfrage bei den Überwachungsbehörden und Giftnotrufzentralen sowie Untersuchung der Prüfzertifikate des TCM-Granulates "Tianwang buxin dan» von 8 in Deutschland verfügbaren Quellen und Analyse dieses Granulates auf Blei, Cadmium und Quecksilber. Ergebnisse: Aktuelle Qualitäts- und Sicherheitsrisiken bestehen in Selbstmedikation, Internethandel, TCM-unerfahrenen Apotheken und bei Granulaten. 11 zwischen 1998 und 2008 publizierte Studien untersuchten die Qualität und Sicherheit von TCA in Deutschland. Auch in den neueren Studien fanden sich teilweise Grenzwertüberschreitungen für Schwermetalle, Pestizide und mikrobiologische Kontaminanten von TCA. Beim Centrum für Therapiesicherheit in der Chinesischen Arzneitherapie (CTCA) gingen in den Jahren 2004-2012 insgesamt 28 Meldungen zu unerwünschten Nebenwirkungen ein. Ein sicherer Zusammenhang wurde in 2 Fällen (allergische Reaktion, Kreislaufreaktion) festgestellt, ein wahrscheinlicher Zusammenhang bestand bei 7 der 28 Meldungen. 61 von 104 (59\%) Zertifikaten von "Tianwang buxin dan" aus 8 Bezugsquellen konnten erhalten werden. 45 der 61 Zertifikate $(74 \%)$ entsprachen den Anforderungen nach der Apothekenbetriebsordnung. Mängel bestanden unter anderem in fehlenden Testungen (Identität, Aflatoxine, Mikrobiologie, Pestizide). Grenzwerte wurden weder in den Zertifikaten noch in den von uns veranlassten Analysen überschritten. Schlussfolgerung: Qualitätsmängel und Sicherheitsrisiken von TCA bestehen weiterhin, müssen allerdings differenziert betrachtet werden. Meldungen zu Nebenwirkungen sind sehr selten.

\author{
Keywords \\ TCM · Expert · Systematic review . \\ Unexpected adverse event - Heavy metal · Pesticide . \\ Aflatoxin
}

\section{Summary}

Quality and Safety of Chinese Medicine Drugs in

Germany - an Update

Background: In the past, traditional Chinese medicine (TCM) drugs (TCD) had in part severe shortcomings regarding quality and safety. The current state of knowledge in this field was evaluated. Material and Methods: Expert interviews by using standardized questionnaires, systematic literature research of studies from Germany published in 1998-2013, systematic inquiries to surveillance authorities and information centers for intoxications as well as investigation of certificates of analysis of the TCM granulate 'Tianwang buxin dan' from 8 available sources in Germany and analyses of its content of lead, cadmium, and mercury. Results: Current quality and safety risks comprise self-medication, TCM-unexperienced pharmacies, Internet sale and granulates. 11 studies published between 1998 and 2008 evaluated the quality and safety of TCD in Germany. Also in newer studies, the values found for heavy metals, pesticides, and microbiological contaminants of TCD in part exceeded the maximum permissible values. 28 reports of adverse events were made to the Center for Therapy Safety in Chinese Drug Therapy (CTCA) within the years 2004-2012. A definite causality was determined in 2 cases (allergic reaction, reaction of the circulatory system); 7 of the 28 reports had a likely causality. 61 of $104(59 \%)$ certificates of 'Tianwang buxin dan' from 8 different sources could be obtained. 45 of 61 certificates $(74 \%)$ complied with the requirements according to the pharmacy work rules. Shortcomings were, amongst others, lacking analyses (identity, aflatoxines, microbiology, pesticides). Critical values were exceeded neither in the certificates nor in the analyses arranged by us. Conclusion: Shortcomings in quality and safety risks of TCD still remain, but must be further distinguished. Reports of adverse events are very rare.

\section{KARGER \\ Fax +49 7614520714

\section{(C) 2014 S. Karger AG, Freiburg}

1661-4119/14/0216-0401\$39.50/0
PD Dr. Roman Huber

Uni-Zentrum Naturheilkunde Freiburg

Breisacher Straße 115B, 79106 Freiburg, Deutschland

roman.huber@uniklinik-freiburg.de 


\section{Einleitung}

Im Jahr 2004 gaben in einer Umfrage in Deutschland 1,6\% der Befragten an, dass sie schon einmal TCA angewendet haben [1]. Der Marktanteil an exportierten TCM-Drogen (TCD) aus China betrug im Jahr 2010 15,8\% für Europa und $3,48 \%$ für Deutschland [2]. Etwa 500 t Drogenmaterial erreichen Deutschland jedes Jahr auf legalem Weg [1]. Im Jahr 2000 bemängelte die Weltgesundheitsorganisation (WHO) die Datenlage zur Sicherheit der TCM, vor allem für Länder außerhalb der Europäischen Union (EU) [3]. Die Situation für Deutschland bezüglich der Qualität und Sicherheit von TCA soll in der vorliegenden Arbeit untersucht werden.

\section{Rechtliche Rahmenbedingungen und Prüfanforderungen}

Ob chinesische Drogen als Lebens- oder Arzneimittel gelten, ist in den Ländern der EU uneinheitlich geregelt. In Deutschland werden TCD bei ihrem Import nicht als Arzneimittel betrachtet und unterliegen daher nicht dem Arzneimittelgesetz (AMG). Diesen Status erhalten sie jedoch, wenn in der Apotheke im Rahmen einer Verordnung verschiedene Drogen zusammengestellt werden. Für die Qualität der TCD und deren Zubereitungen trägt somit der Apotheker die Verantwortung ( $\$ 4$ Abs. 14 und 17 AMG; $§ ~ 7,8$ ApBetrO) [4]. Für jede von der Apotheke abgegebene Droge muss ein Prüfzertifikat vorliegen, das im Normalfall vom Zwischenhändler an die Apotheken mitgeliefert wird. In der Regel wird jede Charge auf ihre Identität und Qualität getestet. Auch wenn die Drogen mit Prüfzertifikat geliefert werden, muss in der Apotheke zumindest eine Identitätsprüfung der bezogenen Ware stattfinden [5]. Anders ist die Situation für Granulate. Da sie beim Import bereits einer Zweckbestimmung unterliegen, werden sie als Arzneimittel betrachtet. Zudem muss der Einführer ein Zertifikat nach § 72a AMG vorlegen, in dem die zuständige Behörde des Herstellungslandes bestätigt hat, dass die Arzneimittel entsprechend den Sicherheits- und Qualitätsstandards der EU hergestellt werden.

Die Qualitäts- und Sicherheitsanforderungen an die TCD und Drogenextrakte sind im Europäischen Arzneibuch (Pharmacopoea Europaea (Ph. Eur.), 7. Auflage, Grundwerk 2011) [6] geregelt. Die aktuelle Ausgabe des Chinesischen Arzneibuches (2010) enthält bereits 2165 Drogenmonographien der TCM, in den vergangenen 5 Jahren wurden über 1000 neue Monographien hinzugefügt [7]. Im Europäischen Arzneibuch sind bereits über 30 Monographien zu chinesischen Arzneipflanzen zu finden und mindestens 20 weitere sind schon fertiggestellt und in Pharmeuropa (www.edqm.eu/en/pharmeuropa-bio-and-scientific-notes-584.html) veröffentlicht.

Die Prüfung bezieht sich auf Identität, Reinheit, Gehalt und unerwünschte Verunreinigungen. Für die Identitätsbestimmung sind neben makroskopischen, sensorischen und mikroskopischen Merkmalen auch physikalisch-chemische sowie chromatographische Methoden vorgesehen. Die Reinheit richtet sich nach der jeweiligen Monographieanforderung und beinhaltet die Bestimmung von Trocknungsverlust, Wassergehalt, Normalasche, säureunlöslicher Asche und fremden Bestandteilen. Auch der Gehalt an ätherischen Ölen oder wirksamen Inhaltsstoffen spielt für die Qualität eine wesentliche Rolle. Unerwünschte Verunreinigungen sind Schwermetalle, Pflanzenschutzmittel (PSM), Mykotoxine und mikrobiologische Kontamination, aber auch die Behandlung mit ionisierender Strahlung, radioaktive Belastung, Restlösemittel bei Extrakten oder toxikologisch bedenkliche Inhaltsstoffe. Für Drogenextrakte ist eine Prüfung aller Aspekte zwar theoretisch möglich, jedoch aufgrund der Schwierigkeit einiger Verfahren bisher nicht routinemäßig durchführbar und somit problematisch. Die Einfuhr von Fertigpräparaten in die EU ohne EU-Zulassung ist seit 2011 verboten. Eine Ausnahme stellt die Einzelanforderung mit ärztlichem Rezept dar, wenn das genannte Präparat im Herstellungsland offiziell zugelassen ist. Fertigpräparate waren in der Vergangenheit für die meisten Sicherheitsrisiken verantwortlich.

\section{Situation in China}

Die Pharmakovigilanz der TCA hat in China in den vergangenen Jahren an Bedeutung gewonnen. Im Jahr 2010 betrafen 13,8\% (2400 Produkte) der gesamten Fallberichte an das Monitoring Center of the Chinese Ministry of Health die TCA; von diesen waren 99,7\% Drogenzubereitungen/Fertigarzneien und $<0,4 \%$ Rohdrogen [8]. Die Mehrzahl der Meldungen zu unerwünschten Arzneimittelwirkungen (UAW) betreffen in China Injektionen aus Drogenextrakten, die in Deutschland keine Anwendung finden [9]. Die TCA-Produktion in China ist mit vielen Problemen behaftet, wie unterschiedliche Saatgutqualität, minderwertige Anbau- und Produktionstechniken, pharmakologisch variierende Qualität bei Wildsammlung, exzessiver Pestizidgebrauch oder Hygienemängel [10], die in neuerer Zeit durch die China Food and Drug Association (CFDA) angegangen werden. Mit der Umsetzung der Good Agricultural and Collection Practice (GACP), die solche Probleme lösen könnte, wurde inzwischen begonnen. Es gibt jedoch bisher keine gesetzlich festgelegten Grenzwerte für Pestizidrückstände oder Schwermetallbelastung [11]. Die aktuelle Ausgabe des Chinesischen Arzneibuches [7] enthält bisher nur Grenzwertangaben für die Drogen Glycyrrhizae radix und Astragali radix [11]. Durch viele kleine Produzenten ist eine durchgängige Qualitätssicherung schwierig und die chargenspezifische Dokumentation kaum möglich. Für einen Drogenimporteur in Deutschland ist es am besten und sichersten, sich Vertragspartner vor Ort zu suchen oder Eigenanbau zu betreiben [1].

\section{Verunreinigung mit Schwermetallen}

Gründe für die Verunreinigung von TCD mit Schwermetallen können umweltverschmutzte Böden durch Industrie oder Düngemittel, eine erhöhte Akkumulationsrate bestimmter Pflanzen [12], falsche Bedingungen beim weiteren Herstellungsprozess (z.B. beim Trocknen, Rösten, Braten usw.) wie 
auch ungünstige Bedingungen beim Lagern oder Verpacken sein. Verschiedene Studien, auch in Europa, haben gezeigt, dass TCD mit Schwermetallen belastet sein können, in einigen Fällen sogar im toxischen Bereich [13]. Zwischen 3 und 75\% der Fertigpräparate hatten einen erhöhten Gehalt der Schwermetalle Blei, Quecksilber oder Arsen [14-17]. In einer aktuellen Studie fanden sich je nach Berechnung bei 1-69\% der Proben gesundheitsschädliche Werte [18]. Außerdem finden sich in Asien und den USA zahlreiche Fallberichte zu Schwermetallintoxikationen im Zusammenhang mit der Verwendung von TCA, wobei die meisten Fälle Fertigpräparate betreffen, die in Asien oder den USA legal erhältlich sind [19-23].

\section{Verunreinigung mit Pestizidrückständen}

In mehreren asiatischen Studien wurden in TCD Verunreinigungen mit PSM gefunden [24-26]. Auch das seit Jahrzehnten verbotene Dichlordiphenyltrichlorethan (DDT) konnte nachgewiesen werden [27]. Bei fehlender Kenntnis über die Entstehungsgeschichte einer Drogencharge muss das ganze Spektrum der Pestizidwirkstoffe untersucht werden, um die Droge in Verkehr zu bringen. Die Kosten dafür sind erheblich. Bei Ware aus dokumentiertem Anbau kann ganz gezielt auf eingesetzte Pestizide untersucht werden bzw. die Rückstandsuntersuchung entfällt, wenn die Anbauflächen nachweislich rückstandsfrei sind, wie dies im deutschen Anbau der Fall ist. In Deutschland sind für Arzneipflanzen keine Pestizide zugelassen.

\section{Mikrobiologie und Mykotoxine}

Mikrobiologische Kontamination ist das nicht beabsichtigte Hinzufügen von infektiösem Material wie Bakterien, Hefen, Schimmelpilzen, Viren, Prionen, Protozoen oder ihrer Toxine oder Nebenprodukte. Auch wenn mikrobiologische Verunreinigungen durch den Kochvorgang gemindert werden, können Bakterien- oder Pilzsporen in TCD verbleiben und gesundheitliche Probleme verursachen. In der Traditionellen Chinesischen Arzneitherapie sind Aflatoxine, wie sie häufig in der klassischen Rezeptur Yuejebaohe Wan gefunden werden, eine bekannte Gefahr [28]. Geregelte Grenzwerte gibt es bisher nur für Aflatoxin B1 $(2 \mu \mathrm{g} / \mathrm{kg})$, für die Summe der Aflatoxine $(4 \mu \mathrm{g} / \mathrm{kg})$, nicht aber für Ochratoxin A [29]. In mehreren, auch europäischen, jedoch nicht TCM-spezifischen Studien konnten Ochratoxine in der Süßholzwurzel (Glycyrrhizae radix) und ihren Produkten nachgewiesen werden [30-32].

\section{Falsche Identifikation}

Ein Beispiel von falscher Identifikation und somit Verwechslung von TCD stammt aus Belgien. Hier wurden Guang Fan Ji (Aristolochia fangchi) und Han Fang Ji (Stephania tetrandra) verwechselt. Die Einnahme der Kapseln, die 100-200 mg Aristolochia fangchi enthielten, als Schlankheitsmittel über einen Zeitraum von 5-20 Monaten führte bei 3-5\% der Patienten zu akutem Nierenversagen [33]. Grund hierfür waren Aristolochiasäuren, die neben ihrer Nephrotoxizität auch kanzerogene Wirkungen haben [34]. Die Drogenmonographien des Chinesischen Arzneibuches sind zur Identitätsbestimmung noch ungenügend, teilweise werden nur mikroskopische Prüfungen verlangt [35]. Methode der Wahl für die Identitätsbestimmung ist die Hochleistungsdünnschichtchromatographie (HPTLC) und für die Gehaltsbestimmung die Hochleistungsflüssigkeitschromatographie (HPLC) [5]. Für die Identitätsbestimmung von Granulaten ist die Methode der ersten Wahl die Dichtechromatographie, ebenso kann die HPLC-Fingerprint-Methode angewendet werden. Nach Schätzungen werden höchstens $30 \%$ aller prüfpflichtigen Substanzen in den Apotheken überprüft. Gründe hierfür sind am ehesten ökonomischer Natur. Jährlich werden etwa 250 Rohdrogen als vermeintlich falsch an die Arzneimittelkommission der Deutschen Apotheker (AMK) geschickt [36].

\section{Zusatz von synthetischen Arzneistoffen}

Der Zusatz von nicht deklarierten chemischen Stoffen ist per definitionem Betrug. Zumeist sind aus dem Internet bezogene, als pflanzliche Nahrungsergänzungsmittel vermarktete, chinesische Fertigarzneien in Form von Pillen oder Salben betroffen [37]. Starke therapeutische Effekte oder schwerwiegende Nebenwirkungen machen auf den Zusatz nicht deklarierter synthetischer Arzneistoffe aufmerksam. Seit dem Verbot der Einfuhr von Fertigarzneien in EU-Länder im Jahr 2011 dürfte für diese nur das Internet als illegale Bezugsquelle ein fortbestehendes Problem darstellen. Nach einer analytischen Studie aus Taiwan von 1997 [38] enthielten 23,7\% von 2609 TCA mindestens einen nicht deklarierten Arzneistoff. Die Liste der zugesetzten Stoffe beinhaltete unter anderem Phenytoin, Glibendamid, Diazepam, Dexamethason, Phenylbutazon, Sildenafil oder Warfarin. In Salben wurden zumeist Corticoide als zugesetzte Stoffe gefunden. Bei Granulaten besteht prinzipiell ebenfalls die Gefahr zugesetzter synthetischer Arzneistoffe; Berichte darüber sind aber bisher nicht bekannt geworden.

\section{Weitere Kontaminanten}

Weitere Kontaminanten in chinesischen Arzneien können Dibenzodioxine und Dibenzofurane, polyzyklische aromatische Kohlenwasserstoffe (PAK), polychlorierte Biphenyle oder Rückstände von Begasungsmitteln wie auch Belastungen mit Fäkalindikatoren sein [5]. PAK können bei der Röstung von Drogen entstehen. Daneben kann bei Hitzeeinwirkung unter Anwesenheit von Zucker auch Acrylamid entstehen [29]. Für Acrylamid sind keine Maximalwerte vorgeschrieben, jedoch gibt es Grenzwerte, bei deren Überschreitung der Hersteller den Produktionsprozess optimieren muss.

\section{Toxikologisch bedenkliche Arzneidrogen}

Bedenkliche Arzneimittel dürfen laut AMG $§ 5$ (1) nicht in den Verkehr gebracht werden. In Deutschland gelten seit 1981 alle Aristolochiasäure-haltigen Arzneimittel als bedenklich 
[39]. Dazu gehören die Pflanzenarten der Gattung Aristolochia, wie beispielsweise die Droge Guang Mu Tong. TCD, die potenziell mit Aristolochiasäure-haltigen Drogen verwechselt werden können, sollten auf Aristolochiasäure getestet sein.

\section{Behandlung mit ionisierenden Strahlen}

Die Bestrahlung mit ionisierenden Strahlen ist eine offizielle Methode im Chinesischen Arzneibuch zur Sterilisation. Für Importeure in die USA oder Australien ist diese sogar zwingend vorgeschrieben. Laut AMG § 7 (1) ist es in Deutschland verboten, pflanzliche Arzneimittel, bei deren Herstellung ionisierende Strahlen verwendet worden sind, in den Verkehr zu bringen. Der starke Rückgang der Kontamination von chinesischen Arzneidrogen mit Keimen könnte auf eine generelle Bestrahlung aller Arzneidrogen in China zurückgeführt werden [1]. Eine Vorschrift zur Testung der Drogen auf Bestrahlung existiert derzeit in Deutschland noch nicht.

\section{Interaktion mit chemischen Medikamenten}

Die Interaktion chinesischer Drogen mit chemischen Medikamenten ist ein noch nicht ausreichend erforschter Bereich. Bekannte Wechselwirkungen gibt es z.B. bei der Verwendung von Radix Salvia miltiorrhizae mit Antikoagulantien oder Thrombozytenaggregationshemmern, da die Droge deren Wirkung verstärken kann.

\section{Fehlende Standards der Verarbeitung und Aufbereitung der Rohdrogen}

Durch verschiedene Verfahren wird die therapeutische Wirkung der TCD verändert oder spezifiziert. Z.B. wird die Eisenhutwurzel (Aconitum carmichaelii) nach längerem Einweichen noch bis zu $6 \mathrm{~h}$ gekocht, bis in der aufgeschnittenen Wurzel kein weißes Zentrum mehr zu erkennen ist. Danach werden die Wurzeln getrocknet. Durch dieses Verfahren entsteht die wesentlich weniger toxische präparierte Eisenhutwurzel (Aconiti radix praeparata). Zudem lassen sich pharmakologische Eigenschaften wie die Geschmacksrichtung, das Temperaturverhalten oder die Wirkungsrichtung ändern. Die chinesischen Heilkräuter können einzeln oder in speziellen Kombinationen geröstet, gedämpft, gebacken oder gekocht werden. Dies kann ohne einen Zusatz oder auch unter Verwendung von Honig, Ingwer, Wein oder Essig geschehen. Sie können außerdem unter Sauerstoffabschluss erhitzt, destilliert oder fermentiert werden. Daneben gibt es noch viele weitere Präparationsverfahren. Wird eine Rohdroge falsch oder ungenügend präpariert, besteht die Gefahr, dass der Anteil an toxischen Inhaltsstoffen zu hoch ist. Somit gibt es einen direkten Zusammenhang zwischen der Verarbeitung, der Qualität und der Sicherheit der chinesischen Arzneien [10, 40].

\section{Falscher Gebrauch}

Unter falschen Gebrauch fallen alle missbräuchlichen, inadäquaten oder falschen Verwendungen der Drogen auf- grund von Unwissenheit, Fahrlässigkeit oder Vorsatz. Ein Großteil der Nebenwirkungen tritt bei Patienten auf, die sich pflanzliche Arzneien selbst verschreiben [41]. Ephedrinhaltiges Ephedrae Herba wurde missbräuchlich in Diätprodukten zur Gewichtsreduzierung verwendet und verursachte Fälle von Vergiftungen, einige von ihnen schwerwiegend [42].

\section{Hepatotoxizität}

Leberschäden sind die Hauptursache für Marktrückrufe von Arzneimitteln generell und betreffen somit auch die TCA. Die bisher untersuchten Fälle waren idiosynkratisch, d.h. nicht dosisabhängig bei Personen mit einer spezifischen Sensibilität gegenüber bestimmten Substanzen. Ein gemeinsames Profil aller betroffenen Personen konnte bislang nicht gefunden werden [43]. Ebenso wenig konnte eine einzelne TCD oder eine Drogenkombination als Ursache identifiziert werden. In der Literatur sind Fälle mit tödlichem Ausgang eines Leberversagens im Zusammenhang mit der Verwendung von TCD beschrieben [9]. Polygonum multiflorum radix enthaltende Präparate [44-47] sowie Dictamnus dasycarpus, Astragalus membranaceus und Paeonia lactiflora tauchten in den verwendeten Rezepturen auf, jedoch konnte keiner dieser Drogen eine direkte hepatotoxische Wirkung nachgewiesen werden [9].

\section{Risiken von Granulaten}

Granulate sind die am häufigsten verwendete TCA-Darreichungsform in Europa. Schätzungsweise 75-80\% aller TCA-Verschreibungen werden in Granulatform eingenommen [48]. Für Deutschland wurden keine Angaben gefunden. Granulate werden aus präparierten Drogen durch Dekoktierung, Eindampfung zur übersättigten Lösung und Aufsprühen dieser auf Hilfsstoffe (z.B. Mais- oder Kartoffelstärke), hergestellt und bestehen aus festen, trockenen Körnern [49].

Bei einer Literaturrecherche wurden keine Daten zur Analyse von Granulaten gefunden, hingegen gibt es zur Analyse von Rohdrogen zahlreiche Literaturangaben [1]. Angaben zu Identitätsprüfungen fehlen gerade bei Granulaten häufig, was das Prüfzertifikat wertlos macht [50]. Nach dem Import nach Deutschland muss die Qualität der Granulate erneut untersucht werden, und für jede Charge muss ein Prüfzertifikat ausgestellt werden. Probleme könnten bei Verunreinigungen der Rohdroge, bei Kontamination durch den Herstellungsprozess, durch den Zusatz von nicht deklarierten Stoffen oder durch Risiken bei der Lagerung der fertigen Produkte (mikrobiologische Aspekte) auftreten. Es gibt europäische Firmen, die zur besseren Qualitätskontrolle und -garantie eigene Plantagen oder zertifizierte Herstellerbetriebe in Asien betreiben $[51,52]$.

Aufgrund der genannten Probleme ist es Ziel der Arbeit, die Informationen zu Qualität und Sicherheit der traditionellen Chinesischen Arzneitherapie in Deutschland zu aktualisieren und wissenschaftlich zu fundieren. 


\section{Material und Methoden}

\section{Expertenbefragung}

Als Einstieg in das Thema wurde eine Expertenbefragung durchgeführt. Die Auswahl der Experten erfolgte auf Vorschlag des Vizepräsidenten der Internationalen Gesellschaft für Chinesische Medizin (SMS) in München. Die Experten waren der erste Sekretär des Centrums für Therapiesicherheit in der Chinesischen Arzneitherapie (CTCA) in Berlin, der Vorstand der Arbeitsgemeinschaft der TCM-Apotheken und ein Mitglied der Europäischen Arzneibuchkommission, Leiter des Instituts für Pharmazeutische Wissenschaften und des TCM-Forschungszentrums der Universität Graz. Die Experten wurden mittels eines Fragebogens hinsichtlich folgender Themen befragt:

- Bedarf an einem Update zur Sicherheitslage von TCD/TCA?

- Wie häufig ist es in den letzten 2 Jahren zu Beanstandungen von TCD in Deutschland gekommen? Wie wurde das dokumentiert?

- Wie sind Reinheit, Identität und Schadstofffreiheit von TCD in Deutschland gesichert?

- In welchem Umfang werden TCD inzwischen in Deutschland angebaut?

- Gibt es Sicherheitslücken im Vertriebssystem? (Internet? Unseriöse Anbieter?) Wenn ja, welche Relevanz haben diese? Wie ist die Verbrauchersicherheit beim Bezug aus dem Internet einzuschätzen?

- Gab es in Deutschland Fälle von Schädigungen/Intoxikationen durch verunreinigte oder falsch deklarierte TCD/TCA?

Die Antworten der Experten auf die Fragen dienten als Grundlage für das weitere Vorgehen bei der Literaturrecherche und Datensammlung.

Am 3. Mai 2013 fand ein von der Bayrischen Landesanstalt für Landwirtschaft organisierter Workshop zum Thema «TCM-Heilpflanzen aus heimischem Anbau: Aktuelle Entwicklungen in Forschung, Produktion und Anwendung» in Freising statt. In diesem Workshop wurden weitere Experten zum Thema Qualität und Sicherheit von TCD befragt.

\section{Literaturrecherche}

Zur Identifizierung von relevanten Quellen wurden die Online-Datenbanken Pubmed (www.ncbi.nlm.nih.gov/pubmed/) und CAM-Base (http://cambase.com/) systematisch durchgesehen. Schlagwörter zur TCM waren «Chinese medicine», «Chinese herbs», «traditional Chinese medicine», «Chinese herbal medicine», «Chinese herbal drugs», «TCM»; zur Qualität der Drogenarzneien «quality», «heavy metals», «lead», «mercury», «cadmium», «toxicity», «toxic», «contamination», «drug contamination», «pesticides», «pesticide residues», «adulteration»; zur Therapiesicherheit «safety», «adverse effects», «adverse reaction», «poisoning», «toxicity», «patient safety», «case report». Hinzu kamen die MeSH-Terms «Medicine, Chinese Traditional» und «Drugs, Chinese Herbal». Eingeschlossen wurden Qualitätsanalysen von für Verbraucher zugänglichen TCA in Deutschland, Fallberichte zu UAW aus Deutschland sowie Studien aus Deutschland zu Nebenwirkungen bei der Anwendung von TCA der letzten 15 Jahre (01.01.1998 bis 31.12.2013). Ausgeschlossen wurden rein pharmakologische Studien, Analysen zu chinesischen Nahrungsmitteln, persönliche Erfahrungsberichte und Fallberichte aus anderen Ländern. Zur Vervollständigung der Recherche wurden von 1998 bis 2013 die Journale arznei-telegramm, Chinesische Medizin (Springer Medizin), Complementary Therapies in Medicine, Deutsche Zeitschrift für Akupunktur, Evidence-Based Complementary and Alternative Medicine, International Journal of Environmental Health Research, Pharmacoepidemiology and Drug Safety, Pharmazeutische Zeitung, Planta Medica, The Journal of Alternative and Complementary Medicine, Zeitschrift für Arznei- \& Gewürzpflanzen und ZTCM Oi-Zeitschrift für Chinesische Medizin systematisch gesichtet. Die Auswahl der Journale erfolgte über die Journale der bereits identifizierten Veröffentlichungen. Des Weiteren wurde eine Literatursammlung des ersten Sekretärs des CTCA bezüglich Qualität zur Identifizierung relevanter Veröffentlichungen genutzt.
Daten von Institutionen zu UAW und Qualitätsmängeln

Um alle Meldungen von UAW der vergangenen 15 Jahre sowie von Arzneimittelbeanstandungen von TCA zu erfassen, wurde systematisch nach Anlaufstellen gesucht. Mit den Suchbegriffen «UAW», «Nebenwirkung», «Meldungen», «unerwünschte Arzneimittelreaktionen» und «TCM» (inklusive verwandter Begriffe) wurde über die Online-Suchmaschine Google nach Anlaufstellen zur Meldung von UAW in Deutschland gesucht. Mit dem Zusatz «TCM» wurde zusätzlich nach Institutionen gesucht, die sich mit der Therapiesicherheit und Qualität von TCM beschäftigen. Identifiziert wurden die Arzneimittelkommission der deutschen Ärzteschaft (Akd̈̈), die AMK, das Bundesinstitut für Arzneimittel und Medizinprodukte (BfArM), das Bundesinstitut für Risikobewertung (BfR), das Bundesamt für Verbraucherschutz und Lebensmittelsicherheit, das CTCA, die Gesellschaft für die Dokumentation von Erfahrungsmaterial der Chinesischen Arzneitherapie (DECA) sowie das «Netzwerk der gegenseitigen Information» vom arznei-telegramm. Diese wurden in Bezug auf UAW bzw. Qualitäts- und Sicherheitsmängel von TCA angeschrieben oder telefonisch kontaktiert.

Weiterhin wurde in der Datenbank der AMK eine Recherche zu UAW von TCA in Auftrag gegeben. Da die Datenbank nach Präparatenamen geordnet ist und eine vollständige Liste von TCA-Präparaten nicht existiert, wurde die Auswahl auf die im Abschnitt «Untersuchung von TCM-Granulaten» genannten Präparate beschränkt. Die Sichtung der Datenbank konnte wegen Personalmangel von der AMK nicht durchgeführt werden.

Darüber hinaus wurden sämtliche Giftinformationszentralen (GIZ) in Deutschland zunächst per E-Mail und, falls keine Rückmeldung erfolgte, telefonisch kontaktiert. Gefragt wurde nach Meldungen zu UAW und Intoxikationsverdachtsfällen von Arzneien jeglicher Art aus dem Bereich der TCM. Die Antwort oder Übermittlung der Daten erfolgte ebenfalls entweder über E-Mail-Kontakt oder telefonisch.

\section{Untersuchung von TCM-Granulaten}

Um möglichst viele Drogen von verschiedenen Anbietern zu untersuchen, wurden die einzelnen Bestandteile der klassischen Rezeptur «Tianwang buxin dan» (deutsch: «Pille des Himmelskönigs zur Stützung des Funktionskreislaufs Herz») in gleich großen Anteilen als Mischung bestellt (Tab. 1).

Mittels Internetrecherche wurden insgesamt 8 mögliche Bezugsquellen für die Rezeptur identifiziert. 7 der 8 Anbieter waren Apotheken, 1 Probe konnte direkt vom Zwischenhändler bezogen werden. Über reine Internetanbieter sind in der Regel nur Fertigarzneien im Sinne von Pillen erhältlich. Die Granulatproben wurden von PhytoLab (Vestenbergsgreuth, Deutschland), einem unabhängigen und zertifizierten Labor, getestet. Die Proben waren einheitlich luftdicht verpackt und mit den Ziffern 1-8 versehen. Der Gehalt der Schwermetalle Blei, Cadmium und Quecksilber wurde mittels Atomabsorption bestimmt. Die Durchführung erfolgte nach der in der Ph. Eur. 7.0 publizierten Methode 2.4.27.

\section{Untersuchung der Prüfzertifikate}

$\mathrm{Zu}$ jedem einzelnen Inhaltsstoff jeder Probe wurden die Prüfzertifikate gemäß $\S 6$ und $\S 11$ der Apothekenbetriebsordnung angefordert. Die Anforderung erfolgte bei Nichterhalt ein zweites Mal. Die Prüfzertifikate wurden nach Form und Inhalt mittels folgender Kriterien beurteilt: Autorisierung zur verantwortlichen Ausstellung, Angaben zu Prüfkriterien, Prüfdatum, Durchführung der Kontaminationsanalysen und eine zusammenfassende Bewertung hinsichtlich der Übereinstimmung mit den Spezifikationen. Die Beurteilung der Prüfzertifikate erfolgte durch N.H.

Jedes Zertifikat muss von einem Sachverständigen nach § 65 AMG unterzeichnet sein, und die Untersuchungen sollten nach pharmazeutischen Regeln sowie vollständig durchgeführt worden sein. Die Vollständigkeit wird dadurch erkannt, dass neben den eigentlich durchgeführten Untersuchungsparametern auch jene aufgeführt sind, die nicht durchgeführt werden konnten. Eine kurze Begründung sollte angegeben sein. 
Tab. 1. Drogenbestandteile der Granulatrezeptur «Tianwang buxin dan»

\begin{tabular}{lll}
\hline Lateinische Bezeichnung & Deutsche Bezeichnung & $\begin{array}{l}\text { Umgangssprachlich } \\
\text { (Pinyin) }\end{array}$ \\
\hline Angelicae sinensis radix & chinesische Engelwurz-Wurzel & $\begin{array}{l}\text { Danggui } \\
\text { Tianmendong }\end{array}$ \\
Asparagi radix & Lebensbaumsamen & Baiziren \\
Biotae semen & Glockenwindenwurzel & Dangshen \\
Codonopsitis radix & Schlangenbartwurzel & Maimendong \\
Ophiopogonis rhizoma/rx & Ballonblumenwurzel & Jiegeng \\
Platycodi radix & Kreuzblumenwurzel & Yuanzhi \\
Polygalae radix & Kokospilzmyzel & Fuling \\
Poria & präparierte Rehmanniawurzel & Shudihuang \\
Rehmanniae radix praep. & Rotwurzelsalbei-Wurzel & Danshen \\
Salvia mil. radix & chinesische Beerentraubenfrüchte & Wuweizi \\
Schisandrae fructus & Ningpo-Braunwurzel & Xuanshen \\
Scrophulariae radix & Stacheljujubensamen & Suanzaoren \\
Zizyphi spinosi semen & & \\
\hline
\end{tabular}

Tab. 2.

Eingeschlossene

Studien in

alphabetischer

Reihenfolge

\begin{tabular}{ll}
\hline Autor, Jahr & Titel \\
\hline Gasser und Zhong, 2004 [53] & $\begin{array}{l}\text { Problematische Drogen: Pheretima (syn. Lumbricus) - Regenwurm } \\
\text { Deuberger et al., 2008 [60] }\end{array}$ \\
& $\begin{array}{l}\text { Dergenqualität chinesischer Heilpflanzen aus bayerischem Versuchsanbau im } \\
\text { Inhaltsstoffe und Reinheit }\end{array}$ \\
Ihrig und Ali, 2001 [54] & Qualität von Drogen der traditionellen chinesischen Medizin \\
Ihrig et al., 2004 [55] & Qualitätsmängel bei TCM-Drogen \\
Krumme et al., 2001 [56] & $\begin{array}{l}\text { Reversible Fanconi syndrome after ingestion of a Chinese herbal 'remedy' containing } \\
\text { aristolochic acid }\end{array}$ \\
Melchart et al., 1998 [61] & $\begin{array}{l}\text { Tolerance of and compliance with traditional drug therapy among patients } \\
\text { in a hospital for Chinese medicine in Germany }\end{array}$ \\
Melchart et al., 1999 [62] & $\begin{array}{l}\text { Monitoring of liver enzymes in patients treated with traditional Chinese drugs } \\
\text { Melchart et al., 1999 [63] }\end{array}$ \\
Melchart et al., 2001 [64] & $\begin{array}{l}\text { Quality assurance and evaluation of Chinese medicinal drugs in a hospital of traditional } \\
\text { Chinese medicine in Germany } \\
\text { 'Quality profiling' for complementary medicine: the example of a hospital for }\end{array}$ \\
Melchart et al., 2003 [65] & traditional Chinese medicine \\
Müller et al., 2009 [57] & $\begin{array}{l}\text { Chinese slimming capsules containing sibutramine sold over the Internet: } \\
\text { a case series }\end{array}$ \\
\hline
\end{tabular}

Ideal wäre es, bei Überschreitung eines oder mehrerer Schwermetallrichtwerte eine Untersuchung zusätzlich aus dem Dekokt beizufügen, um die Unbedenklichkeit der Anwendung zu dokumentieren [53].

\section{Ergebnisse}

Die 6 mittels Fragebogen und Telefoninterview gestellten Fragen wurden von den Experten wie folgt beantwortet:

- Einstimmig wurde Bedarf zur Aktualisierung des Themas «Qualität und Sicherheit von TCD/TCA in Deutschland» gesehen.

- Das CTCA sammelt Meldungen zu UAW.

Reinheit, Identität und Schadstofffreiheit von TCA ist nur für Rohdrogen und Granulate durch Zertifikate gesichert. Die Good Manufacturing Practice (GMP)-Richtlinien für Fertigarzneien wurden als nicht ausreichend erachtet.

- Bei der Frage nach dem Umfang des Anbaus von TCD in Deutschland wurde auf das Projekt der Bayrischen Lan- desanstalt für Landwirtschaft verwiesen (siehe unten). Sicherheitslücken im Vertriebssystem wurden bei Granulaten gesehen, die, wenn sie einmal in Europa sind, unkontrolliert innerhalb der EU gehandelt werden können. Da es in Deutschland teilweise strengere Einfuhrbeschränkungen als z.B. in den Niederlanden oder Belgien gebe, könnten deutsche Sicherheitsstandards nicht garantiert werden.

- Zudem wurde eine Sicherheitslücke in den «nicht spezialisierten» Apotheken gesehen. Wegen zum Teil mangelnder Sachkenntnis über TCM-Arzneien bestünde dort z.B. das Risiko von Verwechslungen oder falschen Dosierungen einzelner Drogen. Außerdem wurde das Risiko durch unseriöse Anbieter im Internet genannt.

- Keiner der Experten konnte Angaben zu aktuellen Fällen von Schädigungen oder Intoxikationen durch verunreinigte oder falsch deklarierte TCA machen.

Auf dem Workshop am 03.05.2013 war zu erfahren, dass seit 1999 mehr als 20 verschiedene TCM-Pflanzen angebaut 
Tab. 3. tudien zur Schwermetallkontamination von Arzneimitteln (TCA) der traditionellen chinesischen Medizin (TCM), sortiert nach der Größe des Probenumfangs

\begin{tabular}{|c|c|c|c|c|c|c|}
\hline \multirow[t]{2}{*}{ Studie } & \multirow[t]{2}{*}{ Probenumfang } & \multicolumn{3}{|c|}{ Grenzwertüberschreitung } & \multirow[t]{2}{*}{ Grenzwertangaben nach } & \multirow[t]{2}{*}{ Bemerkung } \\
\hline & & $\mathrm{Pb}$ & $\mathrm{Hg}$ & $\mathrm{Cd}$ & & \\
\hline $\begin{array}{l}\text { Melchart et al., } \\
2001[64]\end{array}$ & 614 & $3,5 \%$ & & & Deutsches Arzneibuch & $\begin{array}{l}\text { Testung von Rohdrogen; keine } \\
\text { Angaben zu den untersuchten } \\
\text { Drogenarten oder den Konzen- } \\
\text { trationen der einzelnen Schwer- } \\
\text { metalle; keine Informationen zur } \\
\text { Bezugsquelle. }\end{array}$ \\
\hline $\begin{array}{l}\text { Heuberger et al., } \\
2008[60]\end{array}$ & $\begin{array}{l}100 \text { Versuchsproben } \\
\text { (Anbau in } \\
\text { Deutschland) }\end{array}$ & $5-6 \%$ & $3 \%$ & ca. $20 \%$ & $\begin{array}{l}\text { Kontaminantenempfehlungen } \\
\text { (Tab. 1) }\end{array}$ & $\begin{array}{l}\text { Handelsproben zufällig von } \\
\text { verschiedenen Apotheken bezo- } \\
\text { gen; ein Teil der Drogen mit } \\
\text { Überschreitung der Grenzwerte } \\
\text { für Cadmium gelten als Akkumu- } \\
\text { latoren; die Werte sind in der } \\
\text { Originalpublikation als Mittel- } \\
\text { werte der jeweiligen Drogenart } \\
\text { aufgeführt. }\end{array}$ \\
\hline $\begin{array}{l}\text { Ihrig et al., } \\
2004 \text { [55] }\end{array}$ & 42 & $11,9 \%$ & $9,5 \%$ & $23,8 \%$ & $\begin{array}{l}\text { DAC (Deutscher } \\
\text { Arzneimittel-Codex); } \\
\text { entspricht der Kontaminanten- } \\
\text { empfehlung) }\end{array}$ & $\begin{array}{l}\text { Der Bezug der Rohdrogen er- } \\
\text { folgte zum Teil über Apotheken } \\
\text { und zum Teil wurden dem ZL } \\
\text { (Zentrallaboratorium Deutscher } \\
\text { Apotheker) Drogen von der } \\
\text { AMK vorgelegt, die bereits unter } \\
\text { dem Verdacht standen, Qualitäts- } \\
\text { mängel aufzuweisen; mehr als } \\
60 \% \text { der betroffenen Drogen } \\
\text { gelten als Schwermetall-Akku- } \\
\text { mulatoren (z.B. Pheretima, } \\
\text { Artemisia-Arten). }\end{array}$ \\
\hline $\begin{array}{l}\text { Ihrig und Ali, } \\
\qquad 2001[54]\end{array}$ & 18 & $5 / 18$ & $14 / 18$ & $1 / 18$ & $\begin{array}{l}\text { Kontaminantenempfehlungen } \\
\text { (Tab. 1) }\end{array}$ & $\begin{array}{l}\text { Zwischenhändler konnten bei } \\
\text { erneuter Testung diese Werte } \\
\text { nicht bestätigen; } 2 \text { Rohdrogen } \\
\text { stammen aus dem Direktbezug } \\
\text { aus China, die restlichen Drogen } \\
\text { wurden zufällig über Apotheken } \\
\text { bezogen. }\end{array}$ \\
\hline $\begin{array}{l}\text { Gasser und Zhong, } \\
2004[53]\end{array}$ & $\begin{array}{l}12 \text { Chargen } \\
\text { Rohdrogen, } \\
4 \text { Chargen Granulate }\end{array}$ & \multicolumn{3}{|c|}{$\begin{array}{l}\text { Mittelwerte der Rohdrogen } \\
\text { für alle } 3 \text { Schwermetalle } \\
\text { über den Grenzwerten; } \\
\text { Mittelwerte der Granulate } \\
\text { für Blei und Cadmium über } \\
\text { den Grenzwerten }\end{array}$} & $\begin{array}{l}\text { Kontaminantenempfehlungen } \\
\text { (Tab. 1) }\end{array}$ & $\begin{array}{l}\text { Nur gezielt Pheretima (Regen- } \\
\text { wurm) als Droge getestet; in der } \\
\text { Publikation nur Angabe von } \\
\text { Minimal-, Mittel- und Maximal- } \\
\text { werten. }\end{array}$ \\
\hline
\end{tabular}

werden, die seit 2005 Anwendung in der Praxis finden. Vergleichsstudien zwischen Handelsproben und Versuchsproben von TCD bezüglich Qualität wurden in die Literaturrecherche eingeschlossen.

In Pubmed wurden insgesamt 193 Treffer erzielt. Nach Durchsicht der Abstracts und Prüfung der Ein- und Ausschlusskriterien waren 5 Studien für die Auswertung der Qualität und Sicherheit der TCD in Deutschland geeignet. Durch systematische Durchsicht der genannten Journale konnten weitere 3 und über Referenzen nochmals 3 geeignete Arbeiten gefunden werden, sodass insgesamt 11 Arbeiten, die in Tabelle 2 dargestellt sind, eingeschlossen wurden. Hierunter zählen ein Fallbericht, eine Fallserie, 5 Qualitätsanalysen und 5 klinische Beobachtungsstudien zu UAW. Tabelle 3 zeigt die Untersuchungen zur Verunreinigung mit Schwermetallen, Tabelle 4 die Untersuchungen zu Verunreinigungen mit Pestiziden, Tabelle 5 die Untersuchungen zur mikrobiologischen Kontamination und Tabelle 6 Untersuchungen zu sonstigen Kontaminanten von TCD. Prüfzertifikate wurden in 2 Studien im Hinblick auf formale Kriterien beurteilt [54, 55]. In einer der beiden Studien genügten alle 12 Zertifikate von 2 Firmen den formalen Anforderungen der Apothekenbetriebsordnung nicht [54]. In der anderen Studie entsprachen formal 20 von 44 Prüfzertifikaten nicht den Anforderungen nach der Apothekenbetriebsordnung. 
Tab. 4. Studien zur Pestizidbelastung von Arzneimitteln (TCA) der traditionellen chinesischen Medizin (TCM)

\begin{tabular}{|c|c|c|c|}
\hline Studie & Probenumfang & $\begin{array}{l}\text { Ergebnis (Anteil der Drogen } \\
\text { mit Grenzwertüberschreitung) }\end{array}$ & Bemerkung \\
\hline Heuberger et al., 2008 [60] & 100 & $11 \%$ & $\begin{array}{l}\text { Testung nach } \mathrm{Ph} \text {. Eur.; Testung von insgesamt } 10 \\
\text { verschiedenen Kraut- und Wurzeldrogen; vor allem die } \\
\text { Krautdrogen waren betroffen. }\end{array}$ \\
\hline Ihrig und Ali, 2001 [54] & 18 & $5,5 \%$ (1 Probe) & Testung auf Organochlorpestizide. \\
\hline Ihrig et al., 2004 [55] & 3 & keine Grenzwertüberschreitung & $\begin{array}{l}\text { Testung auf } 19 \text { Organochlorpestizide, } 7 \text { Pyrethroide, } \\
17 \text { Organophosphorpestizide, Alachlor, Brompropylat, } \\
\text { Piperonylbutoxid und Dithiocarbamate. }\end{array}$ \\
\hline
\end{tabular}

Tab. 5. Studien zur mikrobiologischen Kontamination von Arzneimitteln (TCA) der traditionellen chinesischen Medizin (TCM)

\begin{tabular}{|c|c|c|c|}
\hline Studie & Probenumfang & $\begin{array}{l}\text { Ergebnis (Anteil der Drogen } \\
\text { mit Grenzwertüberschreitung) }\end{array}$ & Bemerkung \\
\hline Melchart et al., 2001 [64] & 614 & $4,3 \%$ der Proben & $\begin{array}{l}\text { Untersuchung nach den Vorgaben } \\
\text { des Deutschen Arzneibuchs. }\end{array}$ \\
\hline \multirow[t]{2}{*}{ Heuberger et al., 2008 [60] } & 100 (80) Handelsproben & $12 \%(37,5 \%)$ & \\
\hline & $\begin{array}{l}90(70) \text { Versuchsproben (Anbau in } \\
\text { Deutschland) }\end{array}$ & $9 \%(20 \%)$ & \\
\hline Ihrig et al., 2004 [55] & 10 & $0 \%$ & $\begin{array}{l}\text { Die } 10 \text { Proben waren nicht zu } \\
\text { beanstanden. }\end{array}$ \\
\hline
\end{tabular}

Tab. 6. Studien zu sonstigen Kontaminanten von TCD der traditionellen chinesischen Medizin (TCM)

\begin{tabular}{|c|c|c|c|}
\hline Kontaminante & Studie & $\begin{array}{l}\text { Probenumfang/ } \\
\text { Untersuchte TC-Arznei }\end{array}$ & Ergebnis \\
\hline \multirow[t]{2}{*}{ Mykotoxine } & $\begin{array}{l}\text { Heuberger et al., } \\
2008[60]\end{array}$ & 100 Rohdrogen & $\begin{array}{l}\text { In } 2 / 100 \text { Proben Aflatoxine nachweisbar } \\
\text { (unterhalb Grenzwert); in 33/100 Proben } \\
\text { Ochratoxin A nachweisbar (in } 10 \text { Proben } \\
\text { weit über } 10 \mu \mathrm{g} / \mathrm{kg} \text { ). }\end{array}$ \\
\hline & Ihrig et al., 2004 [55] & 7 Rohdrogen & $\begin{array}{l}2 \text { Proben Biotae Semen mit Grenzwert- } \\
\text { überschreitung für Aflatoxin. }\end{array}$ \\
\hline \multirow[t]{2}{*}{ Aristolochiasäure } & $\begin{array}{l}\text { Ihrig et al., } \\
2004[55]\end{array}$ & keine Angaben & $\begin{array}{l}\text { Nachweis von Aristolochiasäure in einer } \\
\text { Probe (Asari Herba). }\end{array}$ \\
\hline & $\begin{array}{l}\text { Krumme et al., } \\
2001[56]\end{array}$ & $\begin{array}{l}1 \text { TC-Fertigarzneimittel } \\
\text { (Akebia } 14^{\circledR} \text { ) }\end{array}$ & Nachweis von Aristolochiasäuren. \\
\hline Identität & $\begin{array}{l}\text { Melchart et al., } \\
2001[64]\end{array}$ & 614 Rohdrogen & $\begin{array}{l}4,6 \% \text { der Proben entsprachen nicht der } \\
\text { angegebenen Identität. }\end{array}$ \\
\hline $\begin{array}{l}\text { Zusatz nicht } \\
\text { deklarierter } \\
\text { Arzneimittel }\end{array}$ & $\begin{array}{l}\text { Müller et al., } \\
2009[57]\end{array}$ & 4 TC-Fertigarzneimittel & $\begin{array}{l}\text { Nachweis von Sibutramin in nahezu der } \\
\text { doppelten in Deutschland zugelassenen } \\
\text { Dosis in } 4 / 4 \text { Proben. }\end{array}$ \\
\hline
\end{tabular}

UAW wurden in den letzten 15 Jahren für Deutschland in 5 Beobachtungsstudien berichtet, die in Tabelle 7 aufgeführt sind. Die Nebenwirkungen wurden zumeist als leicht klassifiziert, in bis zu 1\% der Fälle kam es zu einem deutlichen Anstieg der Leberwerte.

Darüber hinaus gab es in den letzten 15 Jahren in Deutschland 1 Fallbericht und 1 Fallserie zu UAW durch TCA. In dem Einzelfall wurde ein sekundäres Fanconi-Syndrom nach Einnahme eines Aristolochiasäure-haltigen chinesischen Fertigpräparates (Akebia $14^{\circledR}$ ) mit wahrscheinlichem/möglichem
Zusammenhang berichtet [56]. In der Fallserie wurde von 17 Patienten berichtet, die unter Einnahme des Schlankheitsmittels «Lida Dai Dai Hua» unter anderem über Unwohlsein, Tachykardie, arterielle Hypertonie, Übelkeit und Erbrechen klagten. Die chinesische Fertigarznei enthielt den Appetitzügler Sibutramin in der doppelten der in Deutschland zugelassenen Dosierung.

Die Fallmeldungen an das CTCA sind in Tabelle 8 dargestellt. Ein sicherer Zusammenhang wurde nur in 2 Fällen (allergische Reaktion, Kreislaufreaktion) festgestellt, ein wahr- 
Tab. 7. Nebenwirkungen von Arzneimitteln (TCA) der traditionellen chinesischen Medizin (TCM) in klinischen Beobachtungsstudien in Deutschland, sortiert nach Patientenanzahl

\begin{tabular}{|c|c|c|}
\hline Studie & Patienten, $\mathrm{n}$ & Ergebnis \\
\hline $\begin{array}{l}\text { Melchart et al., } \\
1999[63]\end{array}$ & 1597 & $\begin{array}{l}\text { Leichte Nebenwirkungen wie Blähungen, Übelkeit, Erbrechen, Durchfall, } \\
\text { allergisches Erythem in 2\%; kurzzeitige Erhöhung der Leberenzyme in 0,44\%. }\end{array}$ \\
\hline $\begin{array}{l}\text { Melchart et al., } \\
1999 \text { [62] }\end{array}$ & 1507 & Klinisch relevante Erhöhung der Leberenzyme in $1 \%$. \\
\hline $\begin{array}{l}\text { Melchart et al., } \\
2003[65]\end{array}$ & 1036 & $\begin{array}{l}\text { Leichte Nebenwirkungen wie Blähungen, Übelkeit, Erbrechen, Durchfall, } \\
\text { allergisches Erythem in } 1 \% \text {; ein Patient }(0,1 \%) \text { mit schweren Nebenwirkungen } \\
\text { ( } 28 \text { Tage Durchfall); kurzfristige Erhöhung der Leberenzyme in 3\%, } \\
\text { Erhöhung der Leberenzyme bis auf das 2-Fache in } 1 \% \text {. }\end{array}$ \\
\hline $\begin{array}{l}\text { Melchart et al., } \\
1998 \text { [61] }\end{array}$ & $145(168)$ & Mindestens eine leichte Nebenwirkung in 53\% (21\% Übelkeit, 22\% Durchfall). \\
\hline $\begin{array}{l}\text { Melchart et al., } \\
2001[64]\end{array}$ & keine Angabe & Erhöhung der Leberenzyme in $0,9 \%$. \\
\hline
\end{tabular}

Tab. 8. Fallmeldungen an das Centrum für Therapiesicherheit in der Chinesischen Arzneitherapie (CTCA) von 2004 bis 2012

\begin{tabular}{|c|c|c|c|c|}
\hline Fall & Art & $\begin{array}{l}\text { Zusammenhang mit } \\
\text { chinesischer Arzneitherapie }\end{array}$ & Schweregrad & Ausgang \\
\hline 1 & $\begin{array}{l}\text { Leberenzymerhöhung, starke Übelkeit } \\
\text { und Müdigkeit }\end{array}$ & möglich & mäßig & Normalisierung \\
\hline 2 & $\begin{array}{l}\text { Kreislaufreaktion, retrosternale } \\
\text { Schmerzen, Todesangst }\end{array}$ & sicher & mäßig & ohne Folgen \\
\hline 3 & Ikterus & unwahrscheinlich & mäßig & Normalisierung \\
\hline 4 & Interaktion mit Ciclosporin & wahrscheinlich & mäßig & ohne Folgen \\
\hline 5 & Hepatitis mit Ikterus & wahrscheinlich & schwer & Besserung \\
\hline 6 & Hepatitis mit Ikterus & wahrscheinlich & schwer & Besserung \\
\hline 7 & Leberenzymerhöhung & unwahrscheinlich & leicht & $\begin{array}{l}\text { Wiederanstieg ohne weitere } \\
\text { Anwendung von TCA }\end{array}$ \\
\hline 8 & Hepatitis mit Ikterus & ausgeschlossen & schwer & Besserung \\
\hline 9 & vegetative und psychotische Symptome & nicht sicher beurteilbar & mäßig & $\begin{array}{l}\text { Besserung nach medikamentöser } \\
\text { Behandlung }\end{array}$ \\
\hline 10 & Kreatininanstieg und Blutdruckabfall & als Interaktion möglich & mäßig & leichter Rückgang \\
\hline 11 & Leberenzymerhöhung, Müdigkeit & möglich & leicht & Normalisierung \\
\hline 12 & $\begin{array}{l}\text { Photodermatitis nach äußerlicher } \\
\text { Anwendung und Solariumbesuch }\end{array}$ & nicht sicher beurteilbar & mäßig & nicht bekannt \\
\hline 13 & (Qualitätsmangel) & entfällt & entfällt & entfällt \\
\hline 14 & allergische Reaktion: Zungenbrennen & sicher & leicht & ohne Folgen \\
\hline 15 & Leberenzymerhöhung & nicht sicher beurteilbar & leicht & Besserung \\
\hline 16 & Zwischenblutungen & nicht sicher beurteilbar & mäßig & nicht bekannt \\
\hline 17 & Leberenzymerhöhung & unwahrscheinlich & leicht & Normalisierung \\
\hline 18 & Schüttelfrost, Fieber, Prostataschmerz & unwahrscheinlich & leicht & Normalisierung \\
\hline 19 & Impetigo contagiosa (?) & unwahrscheinlich & mäßig & ohne Folgen \\
\hline 20 & «akneförmige» Pusteln & nicht sicher beurteilbar & mäßig & nicht bekannt \\
\hline 21 & pustulöses Ekzem & möglich & mäßig & Normalisierung \\
\hline 22 & ventrikulärer Bigeminus, Extrasystolie & als Interaktion möglich & mäßig & ohne Folgen \\
\hline 23 & Hypertonus (bei Überdosis) & wahrscheinlich & mäßig & weiter Antihypertonika \\
\hline 24 & Durchfall & wahrscheinlich & leicht & Normalisierung \\
\hline 25 & Durchfall & wahrscheinlich & leicht & Normalisierung \\
\hline 26 & Hepatitis & ausgeschlossen & mäßig & Besserung \\
\hline 27 & wässriger Durchfall & wahrscheinlich & mäßig & Normalisierung \\
\hline 28 & Kreatininerhöhung & unwahrscheinlich & mäßig & unverändert \\
\hline
\end{tabular}

scheinlicher Zusammenhang bestand bei 7 der 28 Meldungen. In die gemeinsame UAW-Datenbank der Akd ̈̈ und des BfArM gehen nur UAW-Meldungen zu zugelassenen Arzneimitteln ein, davon überwiegend solchen mit chemisch definierten Wirkstoffen. $\mathrm{Zu}$ TCA waren keine Meldungen bekannt.
Auch auf die Anfragen beim BfR und beim Bundesamt für Verbraucherschutz und Lebensmittelsicherheit wurde geantwortet, dass in den Datenbanken keine entsprechenden Einträge vorlägen. Beim DECA erfolgt keine UAW-Dokumentation von TCA. Bei den GIZ werden in einem Jahresbericht die 
Intoxikationen nach Noxe und Schweregrad dokumentiert, es gibt jedoch keine spezielle Kategorie für TCD und keine Angaben zur Quelle bei Intoxikationen durch Pflanzen. Von 3 der 9 GIZ wurde trotz wiederholter Nachfrage keine Antwort erhalten. Von 3 GIZ wurde mitgeteilt, dass in den letzten Jahren keine Anfragen wegen Intoxikation durch TCA erfolgten. Von 2 weiteren GIZ wurde mitgeteilt, dass die Recherche, da sie von Hand durchgeführt werden muss, nicht durchgeführt werden kann. In der Falldatenbank der GIZ Erfurt (> 200000 Fälle) wurden 2 entsprechende Anfragen gefunden. Im einen Fall hatte eine weibliche Jugendliche nach Einnahme größerer Mengen «Yang Wei Wan»-Pillen, die über das Internet erhältlich sind, keine Symptome, und es waren keine weiteren Maßnahmen notwendig. Im 2. Fall hatte ein männlicher Erwachsener fälschlicherweise etwa $1 \mathrm{~g}$ der Rohdroge Pinelliae rhizoma (Banxia) in 3 Einzeldosen gegessen, statt diese zuzubereiten. Etwa $3 \mathrm{~h}$ danach traten Übelkeit, Stimmveränderungen und Störungen beim Fixieren auf. Das Präparat enthielt vermutlich unter anderem Ephedrin. Eine Gehaltsbestimmung wurde nicht durchgeführt. Da in der präparierten Form der Pinelliae rhizoma in der Regel nur 0,002-0,003\% Ephedrin enthalten sind, lassen sich die Symptome dadurch nicht eindeutig erklären. Es wurde eine stationäre Überwachung empfohlen, der weitere Verlauf ist nicht bekannt.

Die Analyse der 8 Proben ergab (jeweils in $\mathrm{mg} / \mathrm{kg}$ Granulat) für Blei Konzentrationen von 0,09-0,25, für Cadmium $0,01-0,03$ und für Quecksilber $<0,1$ für alle Proben. In keiner der Proben wurden die Referenzwerte überschritten. Insgesamt konnten 61 von 104 (59\%) notwendigen Zertifikaten erhalten werden, nur von 2 Lieferanten kamen alle erforderlichen 13 Zertifikate, bei 2 Lieferanten kamen trotz mehrfacher Aufforderung keine Zertifikate. 45 der 61 Zertifikate (74\%) entsprachen den Anforderungen nach der Apothekenbetriebsordnung. Grenzwerte wurden bei den getesteten Schwermetallen nicht überschritten. Mängel bestanden in fehlenden Testungen (Identität, Aflatoxine, Mikrobiologie, Pestizide) sowie teilweise fehlenden Unterschriften.

\section{Diskussion}

Wie in der Einleitung aufgeführt hat sich die Pharmakovigilanz in China in den letzten Jahren gebessert. Zwischen 2001 und 2008 findet sich in Studien mit TCA-Handelspräparaten allerdings für Deutschland kein eindeutiger Rückgang der Schwermetallbelastung (Tab. 3), der Pestizidbelastung (Tab. 4) und der mikrobiologischen Kontamination (Tab. 5).

Bei Abgabe über spezialisierte Apotheken und in der Hand von erfahrenen Experten scheinen die TCA sicher zu sein. Im Vergleich zur westlichen Pharmakotherapie gibt es weniger Meldungen bezüglich UAW. Bei Selbstmedikation, Bezug über das Internet bzw. in TCM-unerfahrenen Apotheken bestehen allerdings auch in Deutschland die bekannten Risiken fort, und die Sicherheitslage hat sich nicht gebessert (Tab. 7).
Qualitätsmängel bestehen zudem in nicht den Anforderungen entsprechenden Zertifikaten und einer möglichen Behandlung der TCD mit ionisierenden Strahlen in China, was den Vertrieb in Deutschland verbieten würde. Die unzureichende Prüfung von Granulaten kann zu nicht erkannten Qualitätsmängeln und Sicherheitsrisiken führen. Da sie zumeist aus Dekokten hergestellt werden, werden Granulate in Bezug auf Schwermetalle für unbedenklich gehalten, denn mit der Abkochung und Filtrierung gehen nur 6,35-12,2\% der gesamten Schwermetallmenge in das Dekokt über. Zumeist werden die Dekokte jedoch ohne Filterung hergestellt. In einer anderen Studie konnte durch die Herstellung eines Dekokts der Gehalt an Cadmium und Blei zudem nicht signifikant gesenkt werden, und der Übertritt in Wasser scheint auch von der verwendeten Droge abzuhängen. Ein Risiko der Schwermetallbelastung, wie auch der Pestizid- und mikrobiologischen Belastung, kann daher für importierte Granulate keineswegs ausgeschlossen werden. Daher ist die vorgeschriebene Testung der Produkte in jedem Fall unentbehrlich. Die Grenzwertempfehlungen liegen je nach Kommission in medizinischen Produkten bei 3-10 mg/kg für Blei, 0,3-1 mg für Cadmium und 0,1 mg/kg für Quecksilber. In unserer zufälligen Stichprobe lagen die Gehalte deutlich darunter. Studien mit systematischer Beobachtung zu den Nebenwirkungen von TCA wurden für Deutschland seit 2003 nicht mehr publiziert (Tab. 8). Die Meldungen beim CTCA der letzten Jahre sagen zwar nichts über die absoluten oder relativen Häufigkeiten aus, vom Spektrum her passen sie allerdings gut zu den in Studien berichteten $\mathrm{Ne}$ benwirkungen. Es sind dies insbesondere Leberschäden, Durchfall, allergische Reaktionen und Kreislaufreaktionen. Der höhere Anteil an Leberschäden (10 der 28 Meldungen = $36 \%$ ) mag daher rühren, dass beim CTCA hauptsächlich schwerwiegendere UAW gemeldet werden, während in den Studien auch jede leichte UAW erfasst wurde. Zur Qualitätsverbesserung sind die vollständige Qualitätsprüfung der Importware durch die Zwischenhändler bzw. Apotheker, europäische Arzneibuchmonographien, der kontrollierte und dokumentierte Feldanbau sowie die Umsetzung der GACP und der GMP in den Herstellungsländern notwendig.

\section{Dank}

Die Autoren möchten sich herzlich bei der Karl und Veronica Carstens-Stiftung, den befragten Experten Herrn Dr. med. A. Wiebrecht (CTCA), Herrn P. Kwik (Vorstand der Arbeitsgemeinschaft der TCMApotheken) und Herrn Prof. Dr. R. Bauer (Universität Graz) bedanken. Der weitere Dank gilt Herrn Dr. med. J. Hummelsberger (SMS München) und Herrn Dr. E. Meyer-Buchtela der Engel Apotheke Freiburg für die freundliche Unterstützung.

\section{Disclosure Statement}

Die Autoren geben an, dass kein Interessenskonflikt besteht. 


\section{Literatur}

1 Schäfer C: Handhabung von TCM-Drogen in der Apotheke. Pharm Ztg 2005;30.

2 Top 20 export markets of traditional Chinese medicines 2010. www.cccmhpie.org.cn/Pub/3737/25545. shtml (Zugriff 29.10.14).

3 Word Health Organization (WHO): General Guidelines for Methodologies on Research and Evaluation of Traditional Medicines. Geneva, Word Health Organization, 2000, p 74.

4 OVG Lüneburg 11. Senat, Urteil vom 24.10.2002, 11 LC 207/02 Arzneimittel; Pflanzenteil; arzneiliche Zweckbestimmung. www.rechtsprechung.niedersachsen.de/jportal/portal/page/bsndprod.psml? doc. $i d=$ MWRE008220300\&st $=$ null\&showdoccase $=1$ (Zugriff 29.10.14).

5 Gasser U: Arzneidrogen und TCM-Drogen aus Sicht eines Sachverständigen - regulatorische Anforderungen; in Eurofins GMP Praxisforum, Workshop, Hamburg 2011. www.eurofins.de (Zugriff 29.10.14).

6 Pharmacopoea Europaea: Europäisches Arzneibuch Grundwerk 2011, ed 7. Stuttgart, Deutscher Apotheker Verlag, 2013.

7 Zhong W: Das neue chinesische Arzneibuch Ausgabe 2010 ist erschienen. HerbaSinica Kurier 2010;37.

8 Wang H, Ye X, Gao Q, Wu C, Qian Y, Luo B, Sun $\mathrm{Y}, \mathrm{He}$ J: Pharmacovigilance in traditional Chinese medicine safety surveillance. Pharmacoepidemiol Drug Saf 2009;18:357-361.

7 Shaw D: Toxicological risks of Chinese herbs Planta Med 2010;76:2012-2018.

10 Bauer R, Gasser U, Oettmeier R, Rausch H: Quality of TCM drugs and TCM products - current status in the European Union. Pharmeuropa Readers Trib 2013;3:1-10.

11 Zhang B, Peng Y, Zhang Z, Liu H, Qi Y, Liu S, Xiao P: GAP production of TCM herbs in China. Planta Med 2010;76:1948-1955.

12 Wei S, Zhou Q, Mathews S: A newly found cadmium accumulator - Taraxacum mongolicum. J Hazard Mater 2008;159:544-547.

13 Ernst E, Thompson Coon J: Heavy metals in traditional Chinese medicines: a systematic review. Clin Pharmacol Ther 2001;70:497-504.

14 Ko RJ: Adulterants in Asian patent medicines. N Engl J Med 1998;339:847.

15 Cooper K, Noller B, Connell D, Yu J, Sadler R, Olszowy H, Golding G, Tinggi U, Moore MR, Myers S: Public health risks from heavy metals and metalloids present in traditional Chinese medicines. J Toxicol Environ Health A 2007;70:16941699.

16 Martena MJ, Van Der Wielen JC, Rietjens IM, Klerx WN, De Groot HN, Konings EJ: Monitoring of mercury, arsenic, and lead in traditional Asian herbal preparations on the Dutch market and estimation of associated risks. Food Addit Contam Part A Chem Anal Control Expo Risk Assess 2010; 27:190-205.

17 Garvey GJ, Hahn G, Lee RV, Harbison RD: Heavy metal hazards of Asian traditional remedies. Int J Environ Health Res 2001;11:63-71.

18 Harris ES, Cao S, Littlefield BA, et al.: Heavy metal and pesticide content in commonly prescribed individual raw Chinese herbal medicines. Sci Total Environ 2011;409:4297-4305.

19 Auyeung TW, Chang KK, To CH, Mak A, Szeto ML: Three patients with lead poisoning following use of a Chinese herbal pill. Hong Kong Med J 2002;8:60-62.
Hanjani NM, Fender AB, Mercurio MG: Chronic arsenicism from Chinese herbal medicine. Cutis 2007;80:305-308.

21 Lee JJ, Kim YK, Cho SH, Park KS, Chung IJ, Cho D, Ryang DW, Kim HJ: Hemolytic anemia as a sequela of arsenic intoxication following long-term ingestion of traditional Chinese medicine. J Korean Med Sci 2004;19:127-129.

22 Li AM, Chan MH, Leung TF, Cheung RC, Lam CW, Fok TF: Mercury intoxication presenting with tics. Arch Dis Child 2000;83:174-175.

$23 \mathrm{Wu}$ ML, Deng JF, Lin KP, Tsai WJ: Lead, mercury, and arsenic poisoning due to topical use of traditional Chinese medicines. Am J Med 2013;126: 451-454.

24 Wong TC: A survey of heavy metal and organochlorine pesticide contaminations in commercial Lingzhi products. J Food Drug Anal 2007;15:472-479.

25 Xue J, Hao L, Peng F: Residues of 18 organochlorine pesticides in 30 traditional Chinese medicines. Chemosphere 2008;71:1051-1055.

26 Xue J: Overview on external contamination sources in traditional Chinese medicines. World Sci Technol 2008;10:91-96.

27 Leung KS, Chan K, Chan CL, Lu GH: Systematic evaluation of organochlorine pesticide residues in Chinese materia medica. Phytother Res 2005;19: 514-518.

28 Ting A, Chow Y, Tan W: Microbial and heavy metal contamination in commonly consumed traditional Chinese herbal medicines. J Tradit Chin Med 2013;33:119-124.

29 Heuberger H, Bomme U: Schlussbericht des Forschungsvorhabens: Erste züchterische Bearbeitung und Qualitätsbeurteilung ausgewählter chinesischer Heilpflanzen, die für den Anbau in Deutschland geeignet sind. Freising, Bayerische Landesanstalt für Landwirtschaft, 2008, pp 70-100.

30 Arino A, Herrera M, Estopanan G, Juan T: High levels of ochratoxin A in licorice and derived products. Int J Food Microbiol 2007;114:366-369.

31 Herrera M, Herrera A, Arino A: Estimation of dietary intake of ochratoxin A from liquorice confectionery. Food Chem Toxicol 2009;47:2002-2006.

32 Chen AJ, Huang LF, Wang LZ, Tang D, Cai F, Gao WW: Occurrence of toxigenic fungi in ochratoxin A contaminated liquorice root. Food Addit Contam Part A Chem Anal Control Expo Risk Assess 2011;28:1091-1097.

33 Vanherweghem LJ: Misuse of herbal remedies: the case of an outbreak of terminal renal failure in Belgium (Chinese herbs nephropathy). J Altern Complement Med 1998;4:9-13.

34 Cosyns JP: Aristolochic acid and 'Chinese herbs nephropathy': a review of the evidence to date. Drug Saf 2003;26:33-48.

35 Hempen C-H, Fischer T: Leitfaden Chinesische Phytotherapie, vol 2. Jena, Elsevier Urban \& Fischer, 2007.

36 Zhong W: Identitätsprüfung - eine Zeitbombe in der Apotheke entschärft. HerbaSinica Kurier 2009; 35.

37 Ernst E: Adulteration of Chinese herbal medicines with synthetic drugs: a systematic review. J Intern Med 2002;252:107-113.

38 Huang WF, Wen KC, Hsiao ML: Adulteration by synthetic therapeutic substances of traditional Chinese medicines in Taiwan. J Clin Pharmacol 1997; 37:344-350.

39 Aristolochiasäuren. http://de.wikipedia.org/wiki/ Aristolochias\%C3\%A4ure. (Zugriff 24.11.14).
0 Zhao Z, Liang Z, Chan K, Lu G, Lee EL, Chen H, $\mathrm{Li}$ L: A unique issue in the standardization of Chinese materia medica: processing. Planta Med 2010; 76:1975-1986.

41 Ernst E: Toxic heavy metals and undeclared drugs in Asian herbal medicines. Trends Pharmacol Sci 2002;23:136-139.

42 Lee MK, Cheng BW, Che CT, Hsieh DP: Cytotoxicity assessment of Ma-huang (Ephedra) under different conditions of preparation. Toxicol Sci 2000; $56: 424-430$.

43 Medikamentös toxische Schäden. www.medizin1.ukerlangen.de/e68/e131/e1196/e1049/index_ger.html (Zugriff 29.10.14).

44 But PP, Tomlinson B, Lee KL: Hepatitis related to the Chinese medicine Shou-wu-pian manufactured from Polygonum multiflorum. Vet Hum Toxicol 1996;38:280-282.

45 Park GJ, Mann SP, Ngu MC: Acute hepatitis induced by Shou-Wu-Pian, a herbal product derived from Polygonum multiflorum. J Gastroenterol Hepatol 2001;16:115-117.

46 Panis B, Wong DR, Hooymans PM, De Smet PA, Rosias PP: Recurrent toxic hepatitis in a Caucasian girl related to the use of Shou-Wu-Pian, a Chinese herbal preparation. J Pediatr Gastroenterol Nutr 2005;41:256-258.

47 Mazzanti G, Battinelli L, Daniele C, Mastroianni CM, Lichtner M, Coletta S, Costantini S: New case of acute hepatitis following the consumption of Shou Wu Pian, a Chinese herbal product derived from Polygonum multiflorum. Ann Intern Med 2004;140:W30.

48 Granulate. www.lian-chinaherb.ch/index.cfm? fusea ction=products.12748\&lan=de (Zugriff 29.10.14).

49 Jacobsen S: Arzneiformenlehre kompakt und verständlich. Berlin, Weißensee Verlag, 2010.

50 Zhong W: Immer wieder aktuell: Zertifikat ist nicht gleich Zertifikat - Was sagen uns Qualitätsparameter in Zertifikaten. HerbaSinica Kurier 2012;45.

51 Zhong W: Qualität mit starken Wurzeln. HerbaSinica Kurier 2008;33.

52 Phytocomm. Chinesische Pflanzenextrakte. $w w w$. phytocomm.de.

53 Gasser U, Zhong W: Problematische Drogen: Pheretima (syn. Lumbricus) - Regenwurm. CO'MED. Das Fachmagazin für Complementär-Medizin 2004;9:38-39.

54 Ihrig M, Ali SL: Qualität von Drogen der traditionellen chinesischen Medizin. Pharm Ztg 2001;146: 416-422.

55 Ihrig M, Kaunzinger A, Baumann J, Orbig H, Reising K: Qualitätsmägel bei TCM-Drogen. Pharm Ztg 2004;43:3776-3783.

56 Krumme B, Endmeir R, Vanhaelen M, Walb D: Reversible Fanconi syndrome after ingestion of a Chinese herbal 'remedy' containing aristolochic acid. Nephrol Dial Transplant 2001;16:400-402.

57 Muller D, Weinmann W, Hermanns-Clausen M: Chinese slimming capsules containing sibutramine sold over the Internet: a case series. Dtsch Arztebl Int 2009;106:218-222.

58 Lee SH, Wee IJ: Reduction of toxic heavy metals in traditional Asian herbs by decoction preparation. J Bioprocess Biotech 2012;2:122.

59 Nookabkaew S, Rangkadilok N, Satayavivad J: Determination of trace elements in herbal tea products and their infusions consumed in Thailand. J Agric Food Chem 2006;54:6939-6944. 
60 Heuberger H, Bomme U, Groß J: Inhaltsstoffgehalte ausgewählter Heilpflanzen für die traditionelle chinesische Medizin aus deutschem Versuchsanbau im Vergleich zu Importware aus Asien. Z Arznei Gewürzpflanzen 2008;4:173-181.

61 Melchart D, Hager S, Weidenhammer W, Liao JZ, Söllner C, Linde K: Tolerance of and compliance with traditional drug therapy among patients in a hospital for Chinese medicine in Germany. Int $\mathrm{J}$ Risk Saf Med 1998;11:61-64.
62 Melchart D, Linde K, Hager S, Kaesmayr J, Shaw D, Bauer R, Weidenhammer W: Monitoring of liver enzymes in patients treated with traditional Chinese drugs. Complement Ther Med 1999;7: 208-216.

63 Melchart D, Linde K, Weidenhammer W, Hager S, Liao J, Bauer R, Wagner H: Use of traditional drugs in a hospital of Chinese medicine in Germany. Pharmacoepidemiol Drug Saf 1999;8:115-120.
64 Melchart D, Wagner H, Ernst E: Quality assurance and evaluation of Chinese medicinal drugs in a hospital of traditional Chinese medicine in Germany: a five-year report. Altern Ther Health Med 2001;7:24.

65 Melchart D, Weidenhammer W, Linde K, Saller R: 'Quality profiling' for complementary medicine: the example of a hospital for traditional Chinese medicine. J Altern Complement Med 2003;9:193-206. 